\title{
Conseils d'un père à son fils Augustin-Mathurin Le Coz (1752-1780), pour devenir notaire
}

A father's advice to his son, Augustin-Mathurin Le Coz on how to become a notary

\section{Martine Rouellé}

\section{OpenEdition}

\section{Journals}

Édition électronique

URL : http://journals.openedition.org/abpo/2004

DOI : 10.4000/abpo.2004

ISSN : 2108-6443

\section{Éditeur}

Presses universitaires de Rennes

Édition imprimée

Date de publication : 30 mai 2011

Pagination : 61-81

ISBN : 978-2-7535-1691-5

ISSN : 0399-0826

\section{Référence électronique}

Martine Rouellé, « Conseils d'un père à son fils Augustin-Mathurin Le Coz (1752-1780), pour devenir notaire ", Annales de Bretagne et des Pays de l'Ouest [En ligne], 118-2 | 2011, mis en ligne le 30 juin 2013 , consulté le 19 avril 2019. URL : http://journals.openedition.org/abpo/2004 ; DOI : 10.4000/abpo.2004 


\title{
Conseils d'un père à son fils Augustin-Mathurin Le Coz (1752-1780), pour devenir notaire
}

\author{
Martine RouELLÉ \\ Docteur en histoire Université Bretagne-Sud \\ CERHIO CNRS UMR 6258
}

La vie des notaires nous est parfois connue grâce à leurs livres de raison, notamment ceux d'Antoine-Alexandre Barbier, notaire et vigneron bisontin ${ }^{1}$, d'Etienne Borelly notaire à Nîmes sous Louis XIV ${ }^{2}$ ou de PierrePhilippe Candy notaire à Crémieu en Dauphiné ${ }^{3}$. L'approche est ici différente : c'est à travers la correspondance conservée dans le dossier de sa succession ${ }^{4}$ que la vie d'Augustin-Mathurin Le Coz a été partiellement reconstituée. Les lettres émanent principalement de son père. La première date de décembre 1771, alors qu'Augustin exerce la fonction de clerc chez un procureur de Châteaulin, la dernière de novembre 1778. La nouvelle proximité géographique du père et du fils ainsi qu'une brouille interrompent l'échange de courriers. Les autres correspondances proviennent de ses deux frères, Mathurin et Jean-Marie, ainsi que de confrères. Ces courriers retracent le parcours du jeune homme jusqu'à son installation comme notaire. Les lettres du père révèlent un environnement riche d'enseignements à la fois sur la façon de mener sa carrière, sur les règles de la correspondance, les relations père-fils, les contraintes financières, la maladie mais

1. BARBIER, Antoine-Alexandre Une Famille nombreuse au XVIII siècle : le livre de raison d'Antoine-Alexandre Barbier, notaire et bisontin, 1762-1776, présenté par Maurice Gresset, "Résurgences " Privat, Toulouse, 1981, 180 p.

2. SAUZET, Robert, Le Notaire et son roi. Étienne Borelly (1633-1718), un Nîmois sous Louis XIV, Paris, Plon, 1998, 247 p.

3. FAVIER, René, " Un notable hédoniste à la fin du XVIII siècle. Pierre-Philippe Candy, notaire de Crémieu en Dauphiné " dans : Entre justice et justiciables : les auxiliaires de la justice du Moyen Age au XXe siècle, 2005, Les presses universitaires de Laval (Québec), p. 561-578.

4. Les cotes 6 E 3005 et 6 E 3010 des Archives départementales du Morbihan renferment les titres, correspondances ainsi que le règlement de la succession d'un notaire décédé prématurément à Guémené : Augustin-Mathurin Le Coz. 
aussi sur les rapports à la religion, le début de la guerre d'Indépendance américaine, le fonctionnement de la poste aux lettres...

L'inventaire après décès, la vente publique qui suit, le remboursement des créanciers ne sont pas moins utiles à la compréhension du mode de vie du jeune notaire. Les détails de ces documents ont permis d'ouvrir une réflexion sur l'existence d'un très modeste jeune homme de la fin de l'Ancien Régime.

\section{La famille : la branche maternelle prédominante}

Le mariage des parents d'Augustin-Mathurin Le Coz se déroule à Baud le 7 janvier $1749^{5}$. Michel Le Coz est receveur des devoirs au département de Pont-Scorff, originaire de la paroisse de Trégourez située dans l'évêché de Quimper. Son père a exercé comme garde général et collecteur des Amendes, eaux, bois et forêts de Cornouailles, Léon et Tréguier. La fiancée est MarieAnne Jutard, fille de Maître Mathurin Jutard notaire et procureur à Baud ainsi que sénéchal de la juridiction de Kermorvan, et de Jeanne Puren ${ }^{6}$.

Augustin-Mathurin Le Coz naît le 28 août $1752{ }^{7}$ à Saint-Sauveur de Locminé, son père a quitté Pont-Scorff pour exercer à Locminé. Le parrain est Mathurin Richard, receveur des domaines du roi. Il a deux frères. L'aîné, Marie-Mathurin, que son père appelle Mathurin, se marie à Baud en $1777^{8}$. Il est procureur fiscal de Néant, Quistinic et Melrand. Il s'unit avec Marie-Anne Elisabeth Corbel, fille de noble maître François-Mathurin Corbel, ancien avocat à la cour du parlement et de Françoise Daguillon.

Après le décès de Marie-Anne Jutard, Michel Le Coz s'est remarié à Landerneau avec Georgine Bourdon, elle-même décédée avant qu'il ne vienne s'installer près de son fils et qu'il obtienne un poste de procureur fiscal de la juridiction de Quistinic. Il décède à Inguiniel le 25 août 1789, à 75 ans.

Une étude sur les notaires de l'évêché de Vannes (ou du Morbihan) de la fin de l'Ancien Régime à la Restauration a démontré qu'il existe une forte homogamie socio-professionnelle dans le monde des notaires ${ }^{9}$. L'étude des arbres généalogiques laisse apparaître des liens entre les familles qu'elles soient de Vannes, Auray, Hennebont, Lorient, Pontivy, Baud, Rochefort, La Roche-Bernard, Ploërmel, Josselin... Il n'est pas rare, lors de la convocation des parents à l'occasion d'une tutelle, de voir se réunir quatre à cinq notaires alliés à des degrés divers. La majorité des activités de cette famille

5. Arch. dép. du Morbihan BMS Baud 4 E 10/5.

6. Les décombres du château des Rohan ont été acquis par la famille Jutard-Lannivon en 1843 .

7. Arch. dép. du Morbihan BMS Locminé 4 E 117/5.

8. Arch. dép. du Morbihan BMS Baud 4 E 10/6.

9. Rouellé, Martine, Les Notaires du Morbihan et les réformes du notariat sous la Révolution et l'Empire, thèse de doctorat d'histoire, UBS, sous la direction de Gérard Le Bouëdec, novembre 2008, 3 tomes, 1505 p. 
élargie se situe dans le monde des notaires, procureurs, avocats, juges de paix, receveurs des devoirs... Les alliances de cette famille se font principalement entre Baud et Pontivy.

\section{L'éducation : rigueur et humilité}

\section{"Qui aime son fils se hâte de le châtier ${ }^{10}$ ”}

Les qualificatifs donnés par Michel Le Coz à son fils Augustin ne sont pas tendres. Nous ne comptons pas moins de quatre " coquin ", sept "étourdi ", six " effronté ", sept " menteur " sans omettre "morveux", "qui n'a que de mauvaises qualités ", " vaniteux ", " mauvais exemple pour son jeune frère ", " imposteur ", " altier ", " arrogant ", " sot "... Les avertissements fusent, " menaces de coups de bâtons ", " de coup de fouet ", " de correction ", " de lui faire payer son audace, sa désobéissance, ce qu'il mérite " et, bien sûr, rien ne sera oublié, et on se souviendra de lui jusqu'à le menacer de lui couper un membre.

Le projet d'un père pour son fils réside dans l'espoir de le voir devenir un savant homme. L'enfant devient un fils-objet qui a pour finalité la satisfaction du père "qui ne devrait recevoir que des nouvelles consolantes ${ }^{11}$ ". Michel Le Coz traduit bien ce sentiment en écrivant à son fils : "Les enfans qui veullent donner de la satisfaction a leur pere s'empressent de lui faire connoistre le benefice que leurs talens leur procure, il semble que ce soit pour vous une peine de le dire, pendant qu'il est de vôtre devoir de ne le pas sceller. " Tout don du père doit être payé de retour : " Je me suis epuisé à vous elever et vous ay trop donné, puisque vous n'avez pas esté plus reconnoissant."

Privé de liberté et de volonté, l'enfant n'a d'autres desseins que ceux de son père et tout peut arriver si cette image de fils idéal ne colle pas avec la réalité. Cette exigence du père, cette rigueur pouvaient-elles traduire une certaine forme d'amour? Ces sentiments ne sont-ils pas un marchandage d'estime? L'enfant, le jeune homme n'est pas aimé pour ce qu'il est, mais pour le poste qu'il va occuper, pour les honneurs qu'il peut rapporter à la famille, pour le mariage qu'il va conclure... Si les espoirs sont déçus, ce chantage affectif peut conduire à une parfaite inimitié, à un rejet.

La pression ne s'exerce pas seulement du père vers le fils, mais aussi entre les frères. Plusieurs courriers font état de la mauvaise influence qu'exerce Augustin sur son jeune frère qui se conduit mal. Jamais le père ne remet en question l'éducation qu'il donne à ses fils. L'amour paternel est à l'image de l'amour conjugal : raisonné, maîtrisé. Cette raison contraint

10. Delumeau, Jean et Roche, Daniel (dir.), Histoire des pères et de la paternité, Paris, Larousse, 1990, 477 p. Verset de l'Ecclésiaste cité p. 57.

11. Bernos, Marcel, "Images de l'éducation à travers un cahier de thème latin " dans Le XVII siècle et l'éducation, Colloque de Marseille, supplément au numéro 88 de la revue Marseille, $1^{\text {er }}$ trimestre 1977, 188 p., p. 23-35, voir p. 24. 
parfois le père à employer tous les moyens que son amour et sa sévérité peuvent lui inspirer pour corriger le fils ${ }^{12}$.

Le défaut d'obéissance, le mensonge conduisent à ce qui peut arriver de pire : la honte. Dans le cas d'Augustin, c'est le déshonneur de ne pas tout faire pour satisfaire ses protecteurs, les personnes bien placées socialement qui l'emploient. Si Augustin agit mal, l'opprobre risque de rejaillir sur le père. La réaction de Michel Le Coz est toujours la même : la menace d'une bonne correction pour son " fils indigne ". Le modèle positif est présenté dans les vers du "Portrait de l'honnête homme " qu'il a fait apprendre par cœur à son fils.

Outre tous les défauts mentionnés ci-dessus, Augustin a aussi le tort d'être attiré par le jeu, la boisson et d'avoir tenté de se bagarrer. Michel Le Coz souligne ce que nous savons sur les habitudes festives de la basoche: " prennez garde sur tout de vous abandonner à la boisson car le greffe à de tres frequentes occasion pour en former l'habitude». Quand Augustin a résidé une première fois à Châteaulin il a " fait des connnoissances et vu des jeunes gens derangés que je vous avois deffendu de frequenter; vous allez jouer aux cartes malgré les deffenses et precautions que Mad ${ }^{\text {elle }}$ Jullon avois la bonté d'employer pour vous en empecher, corrigez vous de tous ses deffauts ". Il exige donc, à Châteaulin, une conduite tout opposée à celle qu'il a tenue pendant qu'il demeurait chez M. Jullon, c'est-à-dire aussi régulière que la première a été déréglée. Toutes ces heures perdues ne sont pas consacrées à l'étude. Augustin perd son temps " à la bagatelle ", au jeu où l'on perd son argent mais où l'on fait aussi des rencontres suspectes. «Je n'ignore pas les occasions ou les jeunes gens sons en quelque façon obligés de contribuer à des plaisirs communs, mais ce ne doit estre toutes fois que quand on a bien examiné si la situation et les besoins pressans le permettent car un jeune homme ne sera que plus estimés des honestes gens qu'il prefere son entretien à la folle dépense " ou encore : " Il vaut beaucoup mieux avoir menagé de quoy se norir en attandant place que d'avoir depensé son argent à de vains plaisirs. "

Quant à l'image de la mère, elle est conforme à sa position dans la société de l'époque. Elle est sous l'entière domination de son mari. Nous n'avons aucune lettre de Marie-Jeanne Le Coz à son fils, et aucune trace d'échanges entre le père et le fils au moment du décès de celle-ci. Elle n'apparaît qu'à l'occasion de la présentation des respects d'Augustin à sa mère à la fin de ses missives. Occasion nouvelle pour Michel Le Coz de rappeler vertement à son fils les formules de politesse destinées à sa mère.

Bien que les lettres de Michel Le Coz n'épargnent jamais à Augustin de nombreux reproches, ce dernier termine son envoi du 23 juin 1776 par ces propos : "Je finis en vous priant de me permettre de vous ecrire Vous repéte ce que je vous ay dit dans mes précédantes, il n'est pas pour moi de satisfaction plus grande que Celle de M'informer de vos nouvelles et d'en

12. Ibidem, p. 26. 
aprendre si ma Conduitte meritte encorre quelques reproches faites les Luy. Je ne manquerai pas aussitôt De La Reprimer ", preuve de sa volonté de satisfaire son père.

\section{L'instruction}

Concernant l'éducation, Jacques Cambry, lors de son Voyage dans le Finistère ${ }^{13}$, écrit qu'à Landerneau, la situation est si catastrophique qu'il vaut mieux en plaisanter : "Pas un instituteur, pas une institutrice; un maître de danse y mourut de faim; un maître de musique y mourut de soif ". Augustin n'a certainement pas fréquenté de collège. Le sermonnant pour avoir eu une impossibilité " phisique " de se rendre à l'étude, Michel Le Coz lui rappelle que s'il avait profité de l'éducation qu'il a voulu lui donner, il n'aurait pas perdu de l'argent chez M. le curé de Tremavouezan, ni ailleurs, et qu'il aurait été à même de savoir ce qu'est la physique et n'aurait pas utilisé ce terme sans en connaître le sens. C'est donc dans cette paroisse à proximité de Landerneau qu'Augustin a suivi les cours du curé. Il n'y a cependant pas appris le latin, puisque Michel Le Coz lui dit qu'il ne pourrait pas être avocat n'ayant pas cette connaissance.

\section{Les règles de l'écriture et de l'orthographe}

Michel Le Coz ne manque pas une occasion de donner à son fils des leçons sur les règles épistolaires. Sans doute, en a-t-il appris les usages lors de ses stages à Rennes ${ }^{14}$. Les convenances épistolaires " consistent dans l'art de respenser la distance que mettent entre les individus, l'âge, le sexe, le rang, le pouvoir; de n'oublier jamais ce qu'ils sont, et ce que l'on est, de bien calculer ce qu'on peut leur dire, et ce qu'on doit leur taire ${ }^{15}$ ". Ces considérations n'échappent pas à Michel Le Coz : "Le stile epistolaire dont vous vous servez me fait prevenir que vous avez une grande idée de vous mesme, prennez garde coquin que vous n'irritiez au point de vous attacher à la queue d'un cheval. "

La date peut poser un problème de convenance. Comment doit-on la placer dans une lettre selon la personne à qui l'on s'adresse? Michel Le Coz parle d' "impertinences " car, dans le dernier courrier adressé à son père, il a osé mettre la date en haut. " Tel est la maniere dont vous pouvez user quand vous ecrivez à vôtre egal, mais à un superieur la datte se met absolument au bas de la lettre. Je vous laisse a penser ou vous devez mettre la datte des lettres que vous m'ecrivez. "Quand lui-même écrit à son fils, il

13. CAmBry, Jacques, Voyage dans le Finistère ou état de ce département en 1794 et 1795 , Edition critique avec introduction et commentaire par Dany Guillou-Beuzit, Quimper, Société d'Archéologie du Finistère, 1999, 503 p., voir introduction p. L.

14. On peut également supposer qu'il a trouvé dans les ouvrages de la Bibliothèque bleue ou des ouvrages similaires de quoi instruire son fils.

15. Andries, Lise, Bolleme, Geneviève, La Bibliothèque Bleue, Littérature de colportage, Paris, Robert Laffont, 2003, 1012 p., voir p. 881. 
inscrit la date en haut à droite, de même lorsque Mathurin écrit à Augustin. Lorsque l'avocat Pepion écrit à Augustin le lieu et la date figurent en bas à gauche, c'est aussi le cas des lettres du notaire Le Breton.

La marge doit être proportionnelle à la considération que l'on porte à la personne à qui l'on écrit. Quelle soit petite ou grande, elle permet d'éviter la confusion des signes, car il faut empêcher que les lignes s'entrelacent.

Une belle écriture doit respecter l'usage des grandes et des petites lettres. Il est vrai qu'Augustin abuse des majuscules " lettres frisées " au milieu des phrases :

" J'ai eu beau vous inspirez l'envie d'avoir une belle ecriture, mes soins ont esté superflus, pourquoy ne faîtes vous pas attention en ecrivant de suprimer toutes vos lettres frisées, surtout dans le milieu des lignes on ne doit en mettre que le moins qu'on peut quand on ecrit Dieu, par exemple, les noms de villes \& on les met toujours par de grandes lettres, quand on grafes des ecrits, je scai qu'a lors pour employer du papier on tire les plus qu'on peut de lettre frisees mais quand vous faite la copie d'un contract, une ecriture unie et sans lettres frisées est bien plus parente et plus estimée; les procureurs ne s'embarasseront pas de vôtre ecriture pourvu qu'elle se puisse lire mais vous devez estre jaloux de l'avoir belle puisqu'une belle main et de la sagesse est une tres grande resourse. "

On note ici que l'usage des grandes lettres est parfois poussé à l'extrême afin d'utiliser le plus de feuilles possible et de faire augmenter le coût de l'acte sur papier timbré.

La formule de politesse est également l'objet d'une sévère remontrance puisque Augustin se fait traiter de coquin pour avoir écrit :

"Veillez bien présenter à ma cherre mere le mesme respect avec lequel j'ai l'honneur d'estre

Bien sinserement"

Commentaires du père :

"Comment donc coquin depuis bientost trois ans que vous avez sorti de la maison vous n'avez pas encore appris la manierre dont il faut se servir pour ecrire à un pere c'est ce qui me justifie le peux de progres que vous avez fait dans vôtre etat, qu'entendez vous par le terme de bien sincerement, pretendez vous qu'il vaille ceux du respect et de la soumission qui sont de vôtre devoir, apprenez que quand vous ecrivez à un mauvais sujet comme vous la moindre chose que vous puissiez est bien sinserement. "

On n'écrit pas non plus :

"Vôtre tres humble et tres obéissant serviteur, augustin mathurin le Coz, que veut dire ce terme de serviteur. Vous vous immaginez parler à ceux qui vous donnent actuellement du pain a gaigner dont vous estes sans doute le serviteur mais supprimez ce terme quand vous m'ecrivez et apprenez ce qui doit y supleer."

La leçon est aussi valable pour le frère aîné, puisque Michel Le Coz écrit à Augustin de transmettre à Mathurin qu'il est surpris de la fin de sa 
dernière lettre " puisqu'il la termine par ces mots, votre très humble et très obéissant serviteur Le Coz ".

Quelques années plus tard, en 1776, Augustin utilise une autre formule de politesse :

« J'ay l'honneur d'etre d'un profond respec

Mon très Cher pere

Votre très humble et très obeissant serviteur et soumis fils

Augustin mathurin Le Coz

Veuillez bien faire agréer mes très humbles respects à ma Chere mere. "

Nous avons déjà lu les commentaires sur le «serviteur " mais le respect et la soumission doivent satisfaire les exigences du père.

Quand on écrit à quelqu'un chez qui l'on demeure, il faut finir la lettre par ces mots : avec reconnaissance et respect. Si c'est à un supérieur, on ne doit jamais oublier de dire que l'on a l'honneur d'être d'un profond respect. "Etudiez ces termes les plus choisis la soumission et le respect doivent estre les derniers dont vous vous servez pour un pere. "

La politesse veut qu'on emploie le mot juste en faisant allusion à personne. Augustin a écrit, en parlant de Monsieur Destourelles, que c'est " un fort bon garson ". La réponse est aussi cinglante que d'ordinaire : "Convient-il à un morveux comme vous de traiter de garson ceux chez qui vous avez demeuré, vous estes un drole vis avis d'eux etant pourvu de charge et vos superieurs ainsi tout au moins leur devez vous le respect. "

Bien que Michel Le Coz fasse lui-même de nombreuses fautes d'orthographe, il ne peut s'empêcher de signaler à son fils qu'il ne faut pas hésiter à consulter un dictionnaire. En parlant de M. Le Breton, Augustin Le Coz a mentionné qu'il était : " tros avide d'argent et d'ouvrage [...] " d'où " aller chercher dans vôtre dictionnaire et vous trouvez que tros ne s'ecrit pas comme vous le mettez mais bien par trop ". Outre la lecture de la Coutume et de l'ordonnance qui lui apportera les lumières nécessaires, il préconise de "feuilleter vôtre dictionnaire pour apprendre lortographe ". Trois mots sont encadrés par Michel Le Coz pour bien montrer à son fils son erreur : lantienne, " (beau mot qui merite d'estre renfermé parce qu'on le trouve) ", impruntu " (qui s'ecrit par impromtu et s'applique quand il s'agit d'un ouvrage fait sur le champ, au lieu qu'on se sert du terme ", ainsi que coquinarde " (ce mot s'ecrit par goguenarde) ".

Le 5 mars 1774, Michel Le Coz donne à son fils un résumé de sa pensée. Le propre des gens savants réside dans la charité, à cet égard, il est bon qu'Augustin se rappelle les vers que lui a enseignés son père :

" Donnez attention à tout ce que l'on vous dit

Et n'affectez jamais d'avoir beaucoup d'esprit

Vous avez sans doute oublié ces deux vers et leur signification que j'ay tant pris de peine à vous enseigner comme vous ne serez jamais qu'un ignorant (attendu que sans avoir fait de bonnes etudes personnes ni peut estre habile) voyez combien vous serez toute la vie ecarté des sciences puisque 
vous faites journellement des fautes d'ortographe les plus grossieres et malgré cela vous vous croyez un habile moutardier ${ }^{16}$ parce que vous avez appris à dire que vous soutenez la negative, apprenez, sot que le stile epistolaire demande aucun terme de pratique a moins que ce ne soient un avocat ou procureur qui ecrivent a des clients pour les instruire de la situation de leurs procez et encore faut-il estre assuré que les clients entende les termes car en tout autre cas le stile dont on se sert dans une lettre doit estre naturel, bref et sinsere."

Les nombreux conseils de lecture ont été suivis puisque, lors de l'inventaire après décès, on compte des ouvrages dont beaucoup servent à la pratique comme Les principes du droit françois, Le nouveau commentaire sur la coutume civile, L'instruction au droit françois, La coutume de Bretagne, celles de Duparc, d'Argentré et de Sauvageau, Le style criminel, L'instruction faite aux conventions... D'autres relèvent plutôt de la culture générale tel le Dictionnaire de fables et les volumes de L'histoire de France, ainsi que la Grande maire françoise de $\mathrm{Mr}$ de Restoit [sic], et un dictionnaire en deux volumes. Enfin, des ouvrages à caractère religieux comme La nouvelle journée chrétienne, La perfection du chrétien et Les poèmes de la religion.

\section{La religion : tout pêcheur vit dans la crainte de Dieu}

Dieu est souvent invoqué dans les différents courriers de Michel Le Coz ou des amis d'Augustin. Chaque geste de l'existence doit faire craindre de s'éloigner de l'idéal du bon chrétien qui seul méritera la résurrection finale. Les péchés les plus fréquents d'Augustin sont le mensonge, le jeu et les mauvaises fréquentations, l'insolence. À défaut de le voir subir le châtiment de Dieu, il est certain que Michel Le Coz exercera toute son autorité pour punir son fils comme il le mérite. Un vrai chrétien ne doit jamais mentir car Dieu ne sauverait pas la vie d'un homme qui pratiquerait le mensonge. Il doit se garder de relations douteuses et avoir des mœurs édifiantes.

Il peut être surprenant de voir Michel Le Coz, si enclin à inviter son fils à être un bon chrétien, lui écrire de ne pas venir au pardon de Notre-Damedes-Portes. Ce pardon se déroule à Châteauneuf-du-Faou, l'avant-dernier dimanche d'août. Le pardon désigne à la fois la fête religieuse destinée à obtenir des indulgences ainsi que les réjouissances profanes qui l'accompagnent. C'est sur ce dernier point que portent les injonctions de Michel Le Coz. Les pardons deviennent des rendez-vous de danse et de débauche ${ }^{17}$. Les fidèles y trouvent des occasions pour se divertir en mélangeant fête profane et piété ${ }^{18}$. Augustin a certainement dû dépenser son argent lors des

16. " moutardier " selon le Dictionnaire de l'Académie française : " il se croit le premier moutardier du pape " se dit d'un homme médiocre qui a une grande opinion de lui-même, qui se donne des airs importants.

17. Propos du Père Boschet dans Le Floch, Jean-Louis, Pardon en Finistère, Landerneau, Chrétiens médias, 1988, 24 p., voir p. 6.

18. Lebrun, François, Croyances et cultures dans la France d'Ancien Régime, Paris, Le Seuil, 2001, 304 p., voir p. 154. 
fêtes profanes de ce pardon. On sait qu'il aime les divertissements, comme tout jeune homme, et la perspective d'avoir l'occasion de boire du vin et de rencontrer les jeunes filles ne doit pas être du goût de son père qui le somme de renoncer à venir à Châteauneuf.

\section{La situation financière : toujours précaire}

\section{Les revenus}

On remarque le souci constant, voire obsessionnel, du père de connaître le montant des appointements d'Augustin, de lui rappeler sans cesse de ménager ses pratiques, d'économiser ses hardes, d'éviter les déplacements inutiles, les plaisirs coûteux, de ne pas faire d'avances à ses clients. Quelles raisons évoque-t-il au refus qu'il pose d'avancer de l'argent à Augustin? Le jeune frère Jean-Marie est encore à élever et il n'a pas les moyens de lui donner ce qu'il faut pour l'hiver 1771,

« fouillez actuellement dans vôtre poche pour y chercher les dix ecus que vous m'avez si mal employé, que faites vous des appointemens que vous gaignez, je repons pour vous et suis certain que vous les jouez consequement il ne tient qu'à vous de les menager pour avoir des hardes; vous avez raison en disant que vous ne meritez pas mon oubly, car desque j'auray de l'argent et que je pouray m'absenter j'iray vous prouver que je me souviens de vous en remplissant mon devoir et vous rapellant toutes les circonstances qui vous ont ecartées du vôtre c'est tout ce qui vous pouvez esperer de votre père. LeCoz."

En 1773, Michel Le Coz renonce à aller voir son fils car il n'a toujours pas d'argent à dépenser. En 1774, il donne une indication intéressante sur les appointements qu'il touche:

"L'etalage que vous me faites de vôtre sort est tres inutile car je n'ay que trop de peine à donner a vôtre frere Jean marie ses plus pressans besoins encore ne puis-je parvenir que parce que je me prive de ce qui m'est bien necessaire et en effet les appointemens de 340 \# auxquels joint environ 80 \# de remise ne font que $420^{*}$ par an sur quoy nous sommes quatre voyez si vôtre situation n'est pas plus gracieuse que la nôtre. "

420 livres de revenus annuels font de Michel Le Coz un bourgeois moyen, on peut rapprocher ce chiffre du loyer annuel d'Augustin à Guémené de 90 livres/an.

Alors qu'il est veuf de son second mariage, Michel Le Coz envisage de quitter Landerneau qu'il dit détester. Il souhaite rejoindre son fils à Guémené afin de réduire les dépenses : "Si nous avons le malheur de ne pas nous joindre avant l'hiver tu aura plus de peine et moy je regrettray de depenser seul l'argent qui pouroit nous servir tu sens donc combien il est interessant d'abreger une separation aussi onereuse. " Car, en octobre 1778, la bourse de Michel Le Coz ne contient que le prix de la vente de ses meubles. Il semble qu'il ait prêté à son fils Augustin de quoi rembourser les personnes qui lui avaient avancé de l'argent. 


\section{Se vêtir}

Le souci de se vêtir convenablement est une grande préoccupation du jeune clerc. Dès 1771, dans sa première lettre, le père lui parle des hardes qu'il s'est déterminé à lui accorder. Il lui conseille de les conserver précieusement car il ne sera pas en mesure de lui en fournir d'autres. Concernant les souliers, Augustin se plaint des mauvaises réparations faites sur l'une de ses paires. Son père lui répond que s'il considère que les réparations faites par le cordonnier de la famille ne lui conviennent pas, qu'il aille voir un artisan de Châteaulin. Il est hors de question que son père lui donne une paire de souliers neufs, même si, comme il le dit, Augustin est obligé de porter des sabots. Le 5 septembre, il a reçu une nouvelle paire : il n'est pas possible que 2 mois et 22 jours plus tard les souliers soient hors d'usage, c'est là un mensonge.

Il faut savoir prendre soin de ses affaires, ainsi la culotte grise étant trop défectueuse pour être raccommodée, il peut très bien s'habiller de sa culotte de peau, qui, bien entretenue, peut lui servir plusieurs années. Il est également inutile de venir passer les fêtes de Noël à Landerneau, car le voyage ne ferait qu'user les souliers et les hardes dont il manque. Michel Le Coz se souvient que jeune homme, il est parti travailler comme clerc à Rennes et a fait le voyage à pied, portant ses guenilles sur son dos.

En 1772, une lettre indique qu'Augustin se plaint de manquer de bas. Son père lui répond que ce n'est rien en comparaison d'habit, " si vous n'estes pas en etat d'en gaigner quand les vôtres seront usés vous aurez le plaisir d'aller tout nu ". Cependant, il reconnaît qu'Augustin était dans un état d'indigence lorsqu'il est entré chez M. Jullon. Cette remarque n'a pas pour objet de plaindre Augustin, la compassion n'existe pas chez Michel Le Coz, mais de l'exhorter au respect et à la gratitude vis-à-vis de son bienfaiteur.

Lors de l'inventaire après décès, on dénombre deux douzaines de chemises, vingt-cinq cols, dix-sept mouchoirs, vingt-trois paires de bas. On note également trois habits, neuf vestes, neuf culottes, deux pantalons, une redingote, deux gilets, un beverlay gris, deux paires de gants, deux chapeaux, sans compter les caleçons, les cravates et bien sûr une robe de palais, Les sabots sont oubliés au profit de deux paires de bottines et quatre paires de souliers. Augustin Le Coz par son nouveau statut et la place qu'il occupe dans sa paroisse entre dans la "culture des apparences " où le paraître doit révéler le statut et l'être ${ }^{19}$.

\section{La carrière : prendre la bonne voie}

Michel Le Coz exerce pleinement son autorité paternelle pendant la formation de son fils. Il surveille son travail, lui donne des conseils, écrit

19. Roche, Daniel, La Culture des apparences. Une histoire du vêtement, XVII -XVIII siècle, Paris, Fayard, 1989, 564 p., voir p. 481. 
aux personnes qui l'emploient pour s'enquérir de sa conduite. Augustin subit la double autorité de son père et de son employeur.

Augustin-Mathurin Le Coz quitte le domicile familial en 1770 âgé de 18 ans. Il commence une carrière de clerc tout d'abord chez Monsieur Jullon, procureur à Châteaulin. Pendant les absences de M. Jullon, le rôle du clerc est de répondre aux personnes qui pourraient se présenter et de retranscrire leur demande en des notes exactes afin d'en rendre compte au procureur à son retour.

En juillet 1772, Augustin quitte Monsieur Jullon sans en avoir référé à son père. Celui-ci lui reproche de ne pas avoir laissé ses pratiques en dépôt chez son procureur afin de pouvoir s'habiller de pied en cape. Monsieur Jullon se propose également de participer aux frais vestimentaires si les pratiques n'étaient pas suffisantes " pouviez vous souhaiter un plus galent homme ni une offre plus gracieuse et vous avez eu assez peux de sentimens pour n'avoir pas saisy une occasion aussi avantageuse pour votre entretien ». Son nouvel emploi le conduit chez Jacques Le Breton, notaire royal au bourg d'Edern, qu'il quitte le 17 septembre 1773 .

Les indications données sur le stage chez Le Breton concernent encore une fois les hardes. Les pratiques, comme chez Jullon, lui ont procuré des " nipes " chères et il aurait pu en acheter d'autres s'il avait su ménager ce qu'il a reçu : "Vous vous attendiez peut estre que Me le Breton estoit obligé de supléer à vos folles dépences, erreur, n'y lui ni personne n'auront une pareille complaisance, les clercs qui trouvent dans leurs pratiques les ressourses necessaires à leurs besoins doivent se regarder trop heureux et ne jamais rien mal depenser."

Un différend est vraisemblablement la cause du départ. Maître Le Breton doit se rendre à Briec et donne à son clerc de l'ouvrage afin de diminuer les dossiers en attente. Or, Augustin quitte l'étude pour se rendre également à l'audience de Briec. À son retour, Le Breton le sermonne s'apercevant qu'Augustin n'a rempli que 20 à 25 lignes d'écriture. Au lieu de faire preuve d'humilité, Augustin répond que "si cela ne lui convenoit pas il eut à se pourvoir d'un clerc " et il lui donne son congé. Comble de l'effronterie, Augustin retourne à Briec, où il n'avait rien à faire, et rentre prendre son souper chez Me Le Breton.

Le clerc, comme c'était souvent l'usage, logeait chez le notaire qui l'employait ${ }^{20}$. Au commencement du XIX ${ }^{\mathrm{e}}$ siècle, quelques notaires attachés aux vieilles coutumes, nourrissaient encore leur principal clerc et le recevaient à leur table ${ }^{21}$.

Michel Le Coz rappelle à son fils qu'il n'aurait aucune raison de blâmer sa sortie de chez un quelconque procureur à la seule condition qu'il se soit comporté comme il convient : "On entre de bonne grace partout et on en

20. FoIRET, Faustin, Une Corporation pendant la Révolution (Les notaires), Paris, Champion, 1912, 493 p., voir p. 338.

21. Ibidem, voir p. 351. 
sort de mesme. " Il faut savoir ménager ses relations, car si l'on quitte, dans de mauvaises conditions, quelqu'un chez qui l'on a travaillé, il refusera de faire des recommandations. "Il est bon qu'un clerc voye differentes jurisdictions, mais il ne doit quitter une etude que quand il a fait des progres suffisans pour se mieux placer et toujours avec l'agrément des personnes qu'il quitte. "Si Augustin avait suivi ces principes, il n'y aurait eu aucune raison de quitter Le Breton.

En juillet 1774, Augustin devient commis au greffe de Châteauneuf et secrétaire chez Monsieur Sauvage, avocat en parlement et receveur des consignations des sièges royaux du Châteauneuf et Gourin, sénéchal et seul juge du marquisat de la Roche et baronnie de Luz. Cette situation ne semble guère enchanter son père qui le dit " enfoncé dans le secrétariat jusqu'au col ". Selon lui, il vaudrait mieux être chez un procureur notaire car cette place lui donnerait l'occasion d'acquérir les lumières nécessaires à son état. A contrario, la place de secrétaire ne réside qu'en la copie sous diction et la seule connaissance éventuellement acquise réside dans l'énoncé de la sentence. Les fonctions de secrétaire exigent de garder le secret sur les affaires que l'on traite, or Augustin a failli à cette règle l'année précédente. Si Augustin se permettait de renouveler une faute aussi grave chez M. Sauvage, son père le menace, pas moins, de lui couper un membre.

Le 26 août 1774, Maître Claude-Jean-Marie Sauvage remet à Augustin Le Coz une attestation stipulant qu'il a été son secrétaire pendant un an, période pendant laquelle il s'est comporté avec probité et exactitude. La raison de son départ est donnée à la fois par le brouillon d'une lettre d'Augustin à son père et les commentaires de Michel Le Coz. Le $1^{\mathrm{er}}$ juin, l'avocat Sauvage s'est permis de décacheter une lettre de Michel Le Coz destinée à son fils. Pour quitter $\mathrm{M}^{\mathrm{e}}$ Sauvage, Augustin trouve comme prétexte "le deffaut de capacité ". Le père approuve son départ et cette réponse. C'est à nouveau l'occasion de lui demander quels sont ses appointements, question qu'il a déjà posée sans obtenir de réponse.

Dans ce courrier, il conseille à son fils d'acquérir diverses expériences et le greffe ne peut que retarder son avancement. Des compétences multiples offrent l'opportunité de postuler aux places qui se présenteraient. Le dernier conseil consiste en l'usage de ses appointements en " nipes". Bien vêtu, il pourra aller travailler chez un bon procureur. En effet, il est question d'aller travailler chez $\mathrm{M}^{\mathrm{e}}$ Cormao notaire à Châteaulin. Augustin envisage également de se rapprocher de son frère Mathurin, mais Michel Le Coz le lui déconseille. Celui-ci travaille beaucoup mais n'en est pas plus heureux, "les clercs dans l'Évêché de Vannes sont encore plus mal que dans les pays ou vous estes ". Les propos que vient de tenir Michel Le Coz n'auraient pas dû nous parvenir, en effet il conseille à son fils de déchirer sa lettre afin qu'elle ne tombe pas dans des mains étrangères, ce qui montre le secret qui peut entourer la profession.

Les avantages qu'il y a à travailler chez $\mathrm{M}^{\mathrm{e}}$ Cormao résideraient dans l'avancement dont il a besoin pour sa future carrière et également dans la 
possibilité de gagner de quoi subvenir à ses besoins. Michel Le Coz rappelle à son fils qu'il va avoir 22 ans; s'il consacre son temps libre à son instruction - car le temps perdu ne se rattrape jamais -, grâce au travail et à la sagesse, il parviendra aux affaires, " comme bien d'autres qui n'en savoient pas plus que [lui] il y a quelques années et qui aujourd'huy sont bien dans leurs affaires par la bonne conduite et la grande application ". On a assuré à Michel Le Coz que $\mathrm{M}^{\mathrm{e}}$ Cormao était un modèle pour les jeunes gens, aussi ce nouvel emploi devrait être l'occasion de rattraper les erreurs passées.

Or, tout est remis en cause. Augustin n'ira jamais travailler chez $\mathrm{M}^{\mathrm{e}}$ Cormao. Alors que son père le croit à Châteaulin depuis Pâques, il est encore à Châteauneuf. Michel Le Coz qui a écrit à $\mathrm{M}^{\mathrm{e}}$ Cormao pour approuver les démarches de son fils, lui conseille d'aller voir si la place de clerc est toujours libre. En effet, le clerc de Me Cormao est dans l'attente d'obtenir une charge de notaire, c'est ce qui retarde puis annule l'entrée d'Augustin à l'étude.

Fort heureusement, quand Augustin avait annoncé à M. Sauvage qu'il comptait le quitter, il l'avait fait dans des termes honnêtes et reconnaissants. Ceci lui a permis de garder la place, une fois celle de clerc chez $\mathrm{M}^{\mathrm{e}}$ Cormao perdue. Cette expérience doit servir de leçon à Augustin pour " conserver l'amitié des personnes dont vous aurez mangé le pain ", il faut savoir quitter un endroit dans les mêmes bonnes grâces que l'on y est entré. Il ne faut cependant pas perdre de vue la place de clerc chez $\mathrm{M}^{\mathrm{e}}$ Cormao, car le poste de secrétaire qu'occupe Augustin ne sert pas son avancement dans la profession. L'emploi de clerc est bien plus susceptible d'espérances. En faisant preuve d'application et en ayant une bonne conduite, Augustin peut, comme le clerc précédent, espérer avoir sa propre étude. Michel Le Coz estime que la place de secrétaire n'est qu'une perte de temps.

Le projet d'aller se placer dans l'évêché de Vannes est à nouveau évoqué. Michel Le Coz rappelle le sort peu enviable de Mathurin. Il n'y a aucun avantage à tirer d'être sans cesse à se déplacer. " [...] On depense les petits ménagements qu'on a pu faire, soit par les frais qu'ils occasionnent ou par le deffaut de places qui ne se trouvent pas vaccantes " et pendant ce temps il faut se nourrir. Il est bien plus avantageux de chercher une place à proximité plutôt que de vivre au loin dans l'incertitude. À défaut, on peut envisager d'aller plus loin, mais à condition de ne pas faire de déplacements infructueux.

En septembre 1774, une lettre de Michel Le Coz donne une indication sur cette place : "Je scai que vôtre place actuelle vous fournit plus de pratique que chez un procureur " ce qui est peut-être une des raisons du choix d'Augustin. Mais le père a toujours une vision plus lointaine que son fils qui préfère les plaisirs éphémères. Il espère que son fils aura le bonheur d'être procureur à l'âge de 25 ans, sinon il lui décrit ce qui l'attend parlant comme de spectres de ces "vieux clercs qui par leur inconduite n'ont pu trouver occasion de sortir de leur premier etat ". Augustin ne doit pas oublier qu'il 
peut à tout moment perdre sa place et que dans cette optique il doit penser à économiser pour parer à toutes éventualités. Michel Le Coz termine par ces injonctions : "Les pratiques du greffe vous donneront insensiblement un apas pour y demeurer, mais je vous ordonne expressement de le quitter aussitost que vous aurez gaigné ce qu'il faut pour vous habiller et de vous placer chez un bon procureur..."

Sur les conseils de son père, Augustin quitte le greffe et se place chez un procureur de Plouay. Malheureusement, là aussi il déçoit son père. D'une part, Augustin a quitté Châteauneuf sans être assuré de ce qui l'attendait ailleurs, et d'autre part, il entre chez le procureur Bargain, ignorant que ses parents ne vivaient pas en bonne intelligence avec lui. Dès qu'il apprend son impair, il s'arrange pour quitter son emploi et en octobre se place chez M. de Recusson, avocat à la cour et sénéchal du marquisat de Pont Callec.

Dans les papiers, se trouve un extrait des registres du greffe de la juridiction de Nostang et Kervignac daté du 20 décembre 1774. Il s'agit d'une requête présentée par Maître Augustin-Marie Le Coz pour être admis à la place de procureur. Cette demande a été validée par Adrien-Jules-César de Recusson, seigneur du Ménil, avocat à la cour et sénéchal. Nous ne connaissons pas les raisons qui ont fait qu'Augustin n'a pas obtenu cette place. Aucun courrier n'en parle, mais on peut supposer qu'Augustin alors âgé de 22 ans n'a pas obtenu les dispenses d'âge nécessaires puisqu'il n'avait pas atteint l'âge requis de 25 ans. A-t-il également des difficultés à réunir la somme nécessaire à l'achat des provisions?

En juin 1776, Augustin écrit d'Hennebont, est-ce un brouillon ou un original qui n'a pas été envoyé? Un an et demi sans trace de courrier, ce qui ne veut pas dire qu'il n'y ait pas eu de correspondances, mais il est certain qu'une nouvelle brouille a stoppé les échanges épistolaires. La raison est donnée dès le début de cette missive : "Malgré les Défenses que vous m'avez faits de vous ecrire, j'ose me flatter que vous ne vous formaliserez Pas en Contrevenant à vos ordres Puisque ma Désobéissance n'a pour motifs que le rapport que je vous dois d'une affaire qui vous concerne et que mon frere m'a confiée. "

Les relations reprennent entre le père et le fils, la raison, non négligeable, est la réception d'Augustin-Marie à la place de notaire et procureur au Guémené le $1^{\mathrm{er}}$ août 1778 . Ayant enfin atteint le but que son père s'était fixé, Augustin rentre dans ses bonnes grâces. Celui-ci, qui jusque là commençait sa lettre sans en-tête, débute à présent par un "Mon cher Augustin ".

Augustin devient notaire seigneurial et procureur de Jules-Hercule Prince de Rohan au Guémené. Comment Augustin, qui a tant de mal à assurer le quotidien et dont le père peine à boucler son budget, peut avoir les moyens d'acheter sa charge? Une partie de la réponse vient de son ami Pepion de Tallenne. Le Prince de Guémené, n'exigerait pas de finances des nouveaux officiers qu'il vient de créer. La cérémonie de réception consiste en un repas offert par le nouveau nommé. Michel Le Coz n'ayant pas eu 
d'information à ce sujet pense qu'Augustin a sans doute été exempté de cette obligation. Si ce n'est pas le cas, il demande à Augustin de prier ses confrères d'attendre son arrivée pour faire ce repas. La tradition voulait que les jeunes promus invitent les anciens notaires à un banquet ${ }^{22}$.

Toujours soucieux de la comptabilité de son fils, Michel Le Coz le met en garde de ne pas faire d'avances à sa clientèle :

"Tu me marques avoir douze procédure qui t'ont forcés à des avances ce n'est pas le tout de débourser, il faut connoistre la situation des clients, car les insolvables te mettroient hors d'estat de faire plaisir aux riches, en outre tu peu faire entendre à ceux qui s'adresseront à toy qu'en qualité de nouveau procureur tes facultés ne sont pas assez fortes pour subvenir à toutes les dépenses, par ce moyen tu les engagera à donner des acomptes qu'il faut exactement marquer sur la caderne ${ }^{23}$ de chacun affin de ne les pas oublier. "

\section{La maladie : souvent fatale}

À cette époque, les problèmes de santé sont un souci constant. Les plus fragiles ne résistent pas aux maladies, les premiers touchés sont les femmes en couche, les enfants en bas âge et les vieillards.

En 1774, alors qu'il travaille chez M. Le Breton, Augustin attrape la gale avec les enfants de la maisonnée. Si l'on a cru qu'elle était engendrée par "les relliques des mois de purgation des femmes ", ou par la mauvaise qualité du lait des nourrices ${ }^{24}$, Linné reconnaît l'insecte de la gale vers 1734 1746 et le classe sous le nom d'acarus humanus subcutaneus. Cependant, les causes de la gale restent ignorées de la plupart des médecins, on écrivait encore en 1777 que cette maladie avait une origine interne causée par l'acrimonie du sang ${ }^{25}$. Pour se soigner, son père lui conseille une " ptisanne faite avec la racine de parelle pendant 15 jours ou un mois s'il estoit necessaire, apres lequel tems vous eussiez pillé de la rasinne pour en faire dans du boeur roux, ce qui forme un onguent avec lequel on se frote toutes les jointures et partyes du corps excepté sur l'estomac et le ventre ".

La maladie d'Augustin Le Coz est fort détaillée. L'affection est apparue en janvier 1780, dès le 11 février l'apothicaire Martel commence à faire des préparations sur les prescriptions du docteur Maria qui réside à Pontivy. Le médecin n'a sans doute pas encore vu le malade puisqu'il écrit à Augustin

22. FOIRET, Faustin, Une Corporation ..., op. cit., p. 328.

23. Le mot " caderne " ne figure dans aucun dictionnaire. Ce mot est employé à plusieurs autres occasions, notamment les inventaires après décès lorsque l'on fait le compte des minutes " caderne comprise ". Il s'agit tout simplement de ce que nous appellerions aujourd'hui une chemise cartonnée destinée à rassembler diverses pièces d'un même dossier.

24. Georges Vigarello, Le Propre et le sale. L'Hygiène du corps depuis le Moyen-Âge, Paris, Le Seuil, 1985, 283 p., voir p. 51.

25. Nouveau dictionnaire de médecine et de chirurgie pratiques, illustré de figures intercalées dans le texte, sous la direction du DR JACCOUD, Paris, J.-B. Baillière, publié entre 1864 et 1886 , p. 566. 
Le Coz, le 25 janvier, que sa lettre ne présente " aucun détail sur son état et qu'il a donc un problème pour trouver les moyens de le soulager. Il présume une poitrine anciennement affectée et par conséquent faible ". Il se plaint de ce que Josseaume, le chirurgien, n'ait pas fait un exposé de la maladie et des moyens qu'il a déjà employés. L'interrogatoire du patient ou de son entourage suffisait à poser un diagnostic, les consultations se faisaient par correspondance ${ }^{26}$. Maria regrette de ne pouvoir se déplacer en raison des maladies graves qu'il a sur place, mais il invite Augustin à patienter jusqu'au lundi suivant. On sait qu'à l'automne 1779 une grave épidémie de dysenterie a sévi dans la région. À Guémené en octobre 1779, il n'y avait pas un jour sans enterrement ${ }^{27}$. Le 11 février, Maria lui écrit qu'il lui fait passer des drogues, il ne cache pas que son état est " malheureux " et qu'il est dommage d'avoir perdu un temps précieux. Cependant, la belle saison et les remèdes laissent espérer un mieux. Le 21, Maria s'excuse de ne pas être allé le voir à Guémené comme prévu. Il espère s'y rendre le mardi suivant, mais ne pouvant jurer de l'avenir, il lui fait passer

" un lohoch dont vous ferez usage en en prenant une cuillère de deux en deux heures en mettant un quart d'heure avant vos repas, je trouve également util pour diminuer l'état de suffocation que vous preniez en lavement avant votre souper ensuite en se couchant vous avalerez le petit bol que je vous fait passé dans un peu de confiture ou pomme cuite, si vous ne le pouvez avaler tel qu'il est, je vous envoie aussi un bouteille de sirop dont vous mettrez à votre volonté c'est à dire environ une cuillère dans un verre d'eau de guimauve cette boisson doit vous servir dans le courant du jour nonobstant les trois prises de notre tisante coupée avec le lait».

Un courrier du 25 donne à nouveau un remède à base de cresson et de lait à consommer chaud deux à trois fois par jour. Il préconise également un bouillon à base " d'une vieille volaille avec de l'orge mondé et du riz ". Quant à sa nourriture, elle doit être principalement composée de riz gruau, d'œufs, peu de viande à dîner. Le vin ne sera consommé que coupé, car il porte à la fièvre et à l'insomnie.

Le dernier courrier date du 3 mars. Le malade souffre de coliques persistantes dues à des humeurs âcres causes de tous les accidents qui le travaillent. Visiblement le traitement qui soigne les crachats purulents a un effet désastreux sur les intestins. Maria suppose que le lait contenu dans les traitements tourne à l'acide et donne des douleurs d'entrailles.

L'apothicaire Martel présentera une note de 41 livres 11 sols pour les préparations qu'il a délivrées. Elles se composent de looch blanc, médicament magistral, dont l'apparence se situe entre le sirop et le miel. Le blanc, le plus usité, est employé pour soigner les rhumes et les maladies de poi-

26. François LEBRUN, Se soigner autrefois. Médecins, saints et sorciers aux XVII et XVIII siècle, Éditions du Seuil, 1995, 203 p., voir p. 61.

27. Philippe JaRnoux, Villes et campagnes, économie et déplacements de la population. Guémené 1730-1780, Université de Haute Bretagne, Rennes II, 1982-1983., p. 37. 
trine, pour faciliter l'expectoration ou calmer les douleurs ${ }^{28}$. Douze prises de squine qui est surtout employée pour soigner la syphilis, recommandée pour soigner les maladies de peau, les rhumatismes, la goutte ou l'engorgement des viscères ${ }^{29}$. Douze bols expectorants et balzamique : on prépare avec la guimauve des infusions émollientes et adoucissantes, réputées soulager la toux et l'irritation des bronches ${ }^{30}$. Quatre onces de jujubes : le fruit du jujubier est utile contre les cathares, les toux invétérées et généralement les maladies de poitrine ${ }^{31}$, et autant de dattes ayant la même réputation. Quatre onces syrop de lierre terrestre, pectoral contre le rhume et la bronchite $^{32}$, deux onces tablettes de guimauve, qui ont l'avantage de soulager les maux de poitrine et la dysenterie ${ }^{33}$. Une pilule de cynoglosse, fort souvent employée dans la pratique médicale, elle doit ses propriétés calmantes au castoreum, au safran, et surtout à l'opium, qui entrent dans leur composition ${ }^{34}$. Du beaume de soufre anisé, pour les affections bronchiques ${ }^{35}$, deux onces sucre rosat ${ }^{36}$, sucre blanc clarifié et cuit dans de l'eau de rose et quatre onces racine d'althe ${ }^{37}$. Le traitement pouvait soigner les maladies de poitrine, cependant, arrivé à un certain degré de maladie, la bronchite voire la pneumonie associée à des coliques ne peut céder à ce traitement.

Augustin n'ignore pas l'origine de son mal, puisque dans une lettre adressée à Rousseau datant du 21 janvier 1780, il s'excuse du retard pris pour répondre en écrivant que sa "santé était fort affectée et annoncait une attaque de poulmonie que j'ai essuyé et qui ma rendu fort malade, je ne sent même que très légèrement le sympthome de la convalescence, mon espoir n'a désormais de fondement que sur ma jeunesse et le régime le plus rigoureux".

La maladie est vécue dans ces siècles de foi comme un châtiment divin ${ }^{38}$. Quand on échappe aux maladies, l'espérance de vie reste faible.

28. Antoine-Jacques-Louis JouRDAN, Dictionnaire des sciences médicales par une société de médecins et de chirurgiens, Paris, publié de 1812 à 1822 Panckouke, numérisé Paris, BIUM, 2005, p. 69.

29. Antoine-François-Hypolyte FABRE, Le Dictionnaire des dictionnaires de médecine français [...], rédigé par une société de médecins sous la direction du Dr Fabre, Paris, GermerBaillière. Publié en 1850 (supplément : 1851), p. 263.

30. Balsamique sauvage ou impatiens noli tangere dans : Amédée MASCLEF Atlas des plantes de Frances, Paris, éditions Belin, 1987, 2 tomes.

31. Charles-Joseph PANcKoucKe, Encyclopédie méthodique, Paris, publié entre 1787 et 1830 (série médecine : 1787-1830 - série chirurgie : 1790-1798), p. 738.

32. Amédée Masclef, op. cit., p. 220.

33. Charles-Joseph PANCKOUCKE, Encyclopédie méthodique... op. cit., p. 779.

34. Achille RICHARD, Dictionnaire de médecine ou répertoire général des sciences médicales considérées sous le rapport théorique et pratique, par MM. Adelon, Béclard, Bérard, Biett et al., 30 volumes. $2^{\mathrm{e}}$ édition, Paris, Béchet Jeune et Labé. Publié entre 1832 et 1846, p. 553.

35. Jean Le Rond D'AlemBert et Denis Diderot, Encyclopédie ou dictionnaire raisonné des sciences des arts et des métiers par une société de gens de lettres, Paris, Briansson, MDCCLI.

36. Sucre rosat dans : Encyclopédie ou dictionnaire raisonné des sciences des arts et des métiers, op. cit.

37. Guimauve officinale.

38. François LEBRUN, Se soigner autrefois..., op. cit., p. 11. 
L'adulte atteint en moyenne l'âge de 43 ans, la formule " On meurt jeune, il faut vivre vite " s'applique dans notre étude, tant en considération de la courte vie d'Augustin qu'en celle de son entrée dans la vie active.

\section{Le règlement de la succession : déficitaire}

Augustin décède le 6 avril 1780 âgé de 27 ans à Guémené ${ }^{39}$. Le 7 avril, aucun membre de sa famille ne suit le convoi mortuaire.

Le 11 août 1780, un extrait du registre d'audience de la juridiction et siège principal de la principauté de Guémené signale que Michel Le Coz, père héritier ordino verso après un délai de réflexion de trois mois et quarante jours décide de faire déclarer la succession de son fils vacante conformément au second préalable qui est la multiplicité des affaires engagées dans l'étude de Maître Augustin-Mathurin Le Coz. Un procureur, maître Mathurin Cabellec, est nommé pour dresser les différents mémoires et faire rentrer les sommes dues.

Le 19 août 1780, le greffier de la juridiction et siège principal de la principauté de Guémené, comme c'est l'usage en Bretagne, dresse l'inventaire des biens meubles et autres effets mobiliers dépendants de la succession vacante et abandonnée de feu maître Augustin-Mathurin Le Coz. Son logement se compose de deux pièces au rez-de-chaussée, d'une chambre au premier étage que surplombe un grenier. Il dispose également d'une cave, d'une petite cour et de commodités. Le bail débute au 1er mars 1779 pour une année d'un montant de 90 livres versés à la propriétaire, Eulalie Le Guyader, fille de notaire.

Elle est présente lors de l'inventaire ainsi que noble maître Charles François du Feignat avocat au Parlement, substitut dans la succession vacante d'Augustin. On fait appel à un menuisier et à une tailleuse d'habit pour estimer les biens. L'inventaire commence classiquement par la cuisine, dans la salle on note une table de jeu avec une couverture verte. Les plus côtés des livres sont les douze volumes des Principes du droit français évalués pour 18 livres. Dans l'un des tiroirs du bureau on compte, en argent effectif, 19 livres 4 sols et du papier timbré. Le total de l'inventaire se monte à 545 livres 54 sols.

Le 21 août, à huit heures du matin, débute l'inventaire général des minutes, liasses de procédures, mémoires, crédits et autres titres et papiers qui va durer neuf jours. Le 23 août, on procède au compte des crédits dus pour différentes procédures en tant que procureur. On compte 13 pièces dans les liasses de notariat pour l'année 1778, 43 pièces pour l'année 1779, 9 pièces pour l'année 1780 , la plus courte puisqu'elle débute le 4 janvier et se termine le 2 mars. Le greffier note également une liasse de lettres contenant 70 pièces dans laquelle doivent figurer les lettres qui ont permis de retracer son parcours. Le nombre des minutes de notaire peut paraître

39. Arch. dép. du Morbihan, BMS Locmalo 4 E 113/5. 
insignifiant mais on peut constater, lors de l'inventaire des minutes d'autres notaires, une lente ascension. Au Guémené, les minutes de Le Cloirec se montent à 41 en 1750 pour grimper à 301 vingt ans plus tard, ce qui est remarquable pour cette paroisse, mais il est notaire royal et non seigneurial. Tout dépend du nombre de notaires à exercer dans la même juridiction. A l'ouverture des plaids généraux de 1772, on compte 18 notaires dont 12 au moins travaillent et résident habituellement à Guémené ${ }^{40}$. La population est estimée à 1180 habitants à la capitation de 1777. La pratique de leur fonction ne pouvait être financièrement viable qu'à condition d'avoir une clientèle suffisante. Au moment de la réforme du notariat de 1791, la population du canton est estimée à 4287 habitants, celle du chef-lieu à 1261, ce qui fait estimer le nombre des notaires nécessaires à Guémené à deux ${ }^{41}$.

La vente publique se déroule sur deux jours, le 31 août et le 4 septembre. Parmi les acheteurs, on retrouve $\mathrm{M}^{\text {lle }}$ Le Guyader qui achète deux gobelets de verre, une paire de mouchette, la Journée Chrétienne, trois cols, une veste et un mouchoir. $\mathrm{M}^{\text {me }}$ Debroise emporte une cafetière en fer blanc, une courtepointe et une table de jeu. $\mathrm{M}^{\mathrm{lle}}$ Le Cloarec enchérit pour des écritoires, le Principe de droit et le Commentaire de l'ordonnance civile. Le sieur Lannivon se voit attribuer une table longue, un bureau, et une bibliothèque d'étude. Le sieur Jourdan part avec des chemises, un écritoire, un ouvrage religieux et l'Instruction sur les négociants. On compte également parmi les acheteurs, d'autres notaires : Peuchant, Cattier, Le Louarn, Rousseau.

Le montant total de la vente s'élève à 849 livres 14 sols 3 deniers, soit plus de 300 livres de plus qu'à l'estimation de l'inventaire. Des 849 livres 14 sols 3 deniers le greffier a soustrait, pour les frais dus aux personnes ayant participé à l'inventaire, un total de quatre livres six sols. Reste donc 845 livres 8 sols et 3 deniers.

Les comptes de Du Feigna, économe de la succession, font apparaître cette somme à laquelle s'ajoutent les crédits soit 364 livres 11 sols 10 deniers, la bourse commune du greffe 63 livres 3 sols 6 deniers, ainsi que les différentes procédures délivrées par procès-verbal d'un montant de 345 livres 11 sols 3 deniers. Le total s'élève donc à 1618 livres 14 sols 10 deniers. Une fois ôté ce qui est dû à Du Feigna pour avances et vacations ainsi que différents paiements pour 576 livres 13, il reste 1102 livres 1 sol 13 deniers. Cette somme va servir à rembourser les créanciers. Maria, docteur en médecine à Pontivy demande 78 livres. Josseaume le chirurgien chiffre l'exercice de son art à 83 livres 5 sols. Martel, apothicaire, facture 41 livres 11 sols les différentes potions fournies.

Dans l'état des dettes que fait Augustin-Mathurin Le Coz le 4 janvier 1780 , il mentionne " Je dois à mon frère pour une part aux fins de billet 92 livres ». Dupé réclame 82 livres 15 sous 5 deniers. Joseph-Martial Le Briz, notaire, a prêté 120 livres. Dame Perrine-Renée Richard, veuve de noble

40. Philippe JaRnouX, Villes et campagnes..., op. cit., p. 94.

41. Martine Rouellé, Les réformes du notariat..., op. cit., p. 188. 
$\mathrm{M}^{\mathrm{e}}$ Toussaint de Broise, docteur en médecine, sa parente, lui a avancé 84 livres pour " besoins pressants ». Le 26 avril 1779, Maître Louis Cattier, notaire, consent 240 livres de crédit. Un mois plus tard, soit le 20 mai, JeanMarie Peuchant aide Augustin à subvenir à ses besoins pour 60 livres. Les emprunts pour subvenir aux « besoins pressants " représentent 555 livres, ce qui pourrait, au regard du montant de l'inventaire, correspondre au frais d'installation dans sa nouvelle demeure et ses nouvelles fonctions. Sachant qu'Augustin n'a jamais été doué pour l'économie, a-t-il fait ces emprunts après avoir utilisé l'argent prêté par son père comme le mentionne une lettre du 14 octobre 1778 à d'autres fins? Est-ce la raison de la nouvelle brouille entre le père et le fils qui fera que Michel Le Coz ne viendra pas soutenir son fils dans ses derniers instants?

Les frais de subsistance consistent en pension, loyers, fourniture de pain et de viande, chandelles, thé et une main de papier. Les frais d'enterrement s'élèvent à 86 livres.

Il n'est guère aisé de faire un état clair des différentes créances ainsi qu'un bilan comptable. Cependant, il semble que le montant des dettes se monte à 1372 livres et 17 sous. Si l'on rapproche cette somme du crédit restant, mentionné plus haut, soit 1102 livres 1 sol 13 deniers, la succession est déficitaire d'environ 270 livres.

Augustin est issu d'une famille dans laquelle se croisent les receveurs des devoirs et les notaires. Le premier cercle familial maternel offre à Augustin et son frère Mathurin l'opportunité de faire carrière comme notaire et procureur pour le premier, comme procureur pour le second. Côté paternel, on ne fait mention que d'une tante qui semble peu fortunée, il est donc naturel que les enfants se soient tournés vers les oncles plus solidement installés dans l'évêché de Vannes. Le second cercle composé de la famille plus éloignée et des amis permet aussi de se faire un chemin dans la carrière, voire d'emprunter de l'argent quand cela est nécessaire.

Michel Le Coz vit en bon chrétien, pratique une éducation rigoureuse, prône les vertus du travail et possède un sens aigu de l'économie. Il essaie de transmettre ces qualités à son fils. Si l'enfant a dû recevoir quelques bons coups de bâton pour le pénétrer de ses principes, le jeune adulte est plus difficile à régenter.

L'argent est un bien rare qu'il faut utiliser avec infiniment de discernement afin de se vêtir et d'assurer le quotidien. Toute dépense qui relève du futile est absolument à bannir. Chaque jour doit être consacré à l'étude et à la poursuite d'un but bien précis, être installé professionnellement dès que possible. Malheureusement tout ne peut pas être maîtrisé et, comme beaucoup de ses contemporains, Augustin subit la maladie qui finit par l'emporter à presque 28 ans. Sa mort ne met pas fin à la connaissance que nous pouvons avoir de ce jeune homme, les procédures liées à la succession nourrissent notre quête de compréhension. 
Outre les informations strictement relatives à notre personnage, les courriers font également découvrir le monde qui l'entoure. Les façons de vivre et de penser en Bretagne en cette fin d'Ancien Régime se dessinent au fil des lettres. Ce précieux témoignage a permis de mieux appréhender la vie des notaires à la fin de l'Ancien Régime et de mesurer l'impact de la réforme de 1791, prélude à celle de ventôse an XI.

\section{RÉSUMÉ}

Augustin-Mathurin Le Coz quitte le domicile familial a 18 ans en 1770 . Les correspondances échangées avec son père, receveur des devoirs à Landerneau, font connaître les règles d'éducation données à son fils. Le savoir-vivre le savoir écrire, les rapports à l'argent, le religieux sont autant de thèmes abordés. Les courriers reconstituent également le parcours professionnel et le contexte local. Les autres missives et documents officiels restituent d'autres pans de la vie de ce jeune notaire décédé au Guémené à 27 ans.

\section{ABSTRACT}

Augustin-Mathurin Le Coz left his family home at 18 years old in 1770. His correspondence with his father, a tax collector in Landerneau, reveals the educational guidelines the latter gave to his son. Manners, writing skills, relationship to money, and view of religion were a few of the many topics broached. The letters also help piece together his professional life, as well as the local context. Other letters and official documents show the other aspects of the life of this young notary who died in Guémené at the age of 27. 